\title{
Unilateral traumatic brain injury of the left and right hemisphere produces the left hindlimb response in rats
}

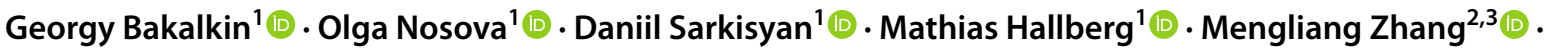 \\ Jens Schouenborg ${ }^{3}$ (i) $\cdot$ Niklas Marklund $^{4}$ (i) $\cdot$ Hiroyuki Watanabe ${ }^{1}$ (I)
}

Received: 9 February 2021 / Accepted: 20 April 2021 / Published online: 22 May 2021

(c) The Author(s) 2021

\begin{abstract}
Traumatic brain injury and stroke result in hemiplegia, hemiparesis, and asymmetry in posture. The effects are mostly contralateral; however, ipsilesional deficits may also develop. We here examined whether ablation brain injury and controlled cortical impact (CCI), a rat model of clinical focal traumatic brain injury, both centered over the left or right sensorimotor cortex, induced hindlimb postural asymmetry (HL-PA) with contralesional or ipsilesional limb flexion. The contralesional hindlimb was flexed after left or right side ablation injury. In contrast, both the left and right CCI unexpectedly produced HL-PA with flexion on left side. The flexion persisted after complete spinal cord transection suggesting that CCI triggered neuroplastic processes in lumbar neural circuits enabling asymmetric muscle contraction. Left limb flexion was exhibited under pentobarbital anesthesia. However, under ketamine anesthesia, the body of the left and right CCI rats bent laterally in the coronal plane to the ipsilesional side suggesting that the left and right injury engaged mirror-symmetrical motor pathways. Thus, the effects of the left and right CCI on HL-PA were not mirror-symmetrical in contrast to those of the ablation brain injury, and to the left and right CCI produced body bending. Ipsilateral effects of the left CCI on HL-PA may be mediated by a lateralized motor pathway that is not affected by the left ablation injury. Alternatively, the left-side-specific neurohormonal mechanism that signals from injured brain to spinal cord may be activated by both the left and right CCI but not by ablation injury.
\end{abstract}

Keywords Traumatic brain injury $\cdot$ Stroke $\cdot$ Ipsilateral response $\cdot$ Contralateral response $\cdot$ Postural asymmetry

$\begin{array}{ll}\text { Abbreviations } \\ \text { ATNR } & \text { Asymmetric tonic neck reflex } \\ \text { CCI } & \text { Controlled cortical impact } \\ \text { HL-PA } & \text { Hindlimb postural asymmetry } \\ \text { MPA } & \text { Magnitude of postural asymmetry } \\ \text { PAFs } & \text { Postural asymmetry inducing factors }\end{array}$

Communicated by Francesco Lacquaniti.

Georgy Bakalkin

Georgy.Bakalkin@farmbio.uu.se

1 Department of Pharmaceutical Biosciences, Uppsala University, Husargatan 3, Box 591, 75124 Uppsala, Sweden

2 Department of Molecular Medicine, University of Southern Denmark, Odense, Denmark

3 Neuronano Research Center, Department of Experimental Medical Science, Lund University, Lund, Sweden

4 Skåne University Hospital, Department of Clinical Sciences Lund, Neurosurgery, Lund University, Lund, Sweden

\section{Introduction}

Traumatic brain injury and stroke result in asymmetric motor deficits including contralesional hemiplegia and hemiparesis and asymmetry in posture and balance (Zhang et al. 2020). Severity of impairments of contralesional limbs may be hemisphere specific and depend on side of brain lesion (Hanna-Pladdy et al. 2002; Harris and Eng 2006; Mani et al. 2013; de Paiva Silva et al. 2018). In right-handed individuals, the contralesional deficits are more severe after right hemisphere damage. Right hemisphere damage by stroke induces poorer muscular responses to stance perturbation and leads to poorer postural responses both in quiet and perturbed balance relative to left cerebral damage (Fernandes et al. 2018; Coelho et al. 2019).

Besides contralesional motor impairments, unilateral brain injuries including traumatic brain injury and stroke in humans and experimental animals result in substantial motor deficits in the ipsilateral half of the body including the ipsilesional limb (Gonzalez et al. 2004; Varghese and 
Winstein 2019; Maenza et al. 2020). Injury to the motor cortex in rats produced severe deficits in skilled reaching in both the contra- and ipsilateral forelimbs, suggesting that skilled movements are under bilateral control by both hemispheres (Gonzalez et al. 2004). In stroke survivors, impairments of the ipsilesional extremities depend on the side of brain lesion and vary with the severity of contralesional deficits (Varghese and Winstein 2019; Maenza et al. 2020). In patients with left hemisphere injury, ipsilesional motor deficits are proportional to the degree of impairments while this relationship is not definite in patients with injury to the right hemisphere. In other words, damage to the left hemisphere produced greater ipsilesional deficits than that to the right hemisphere.

Animal studies demonstrated that a unilateral lesion to the cerebellum and sensorimotor cortex caused asymmetric hindlimb posture (Chamberlain et al. 1963; Zhang et al. 2020), while a lateral spinal cord hemisection produced changes in spinal reflexes that paralleled asymmetry in locomotion (Hultborn and Malmsten 1983; Rossignol and Frigon 2011). Cerebellar or spinal cord injuries produced flexion or activated nociceptive withdrawal reflexes of the ipsilesional hindlimb. In contrast, the contralesional hindlimb was flexed after a localized unilateral focal lesion of the sensorimotor cortex (Lukoyanov et al. 2020; Watanabe et al. 2020) or a large hemispheric injury (Varlinskaia et al. 1984). Formation of hindlimb postural asymmetry (HL-PA) after a cortical lesion is paralleled by impairments of contralesional hindlimb function in the beam walking and ladder rung tests (Lukoyanov et al. 2020) as well in activation of hindlimb nociceptive withdrawal reflexes evoked by electrical stimulation and recorded with EMG technique on the contra- $v s$. ipsilesional side (Zhang et al. 2020). The asymmetry induced by the focal cortical injury was abolished by pancuronium, a curare-mimetic muscle relaxant suggesting a neural mechanism dependent on efferent drive. The changes in posture and reflexes induced by brain injury persisted after complete spinal cord transection suggesting that they were encoded in spinal neurocircuits (Chamberlain et al. 1963; Rossignol and Frigon 2011; Zhang et al. 2020). The HL-PA phenomenon in rats recapitulates several pathophysiological features of the human upper motor neuron syndrome including the "hemiplegic posture" and exaggerated asymmetric withdrawal reflexes and represents a reproducible and quantifiable model to unravel neurobiological mechanisms of sensorimotor and postural deficits.

A translational value of the HL-PA model is supported by the findings that the same phenomenon is induced by the controlled cortical impact (CCI), a clinically relevant focal unilateral traumatic brain injury (Watanabe et al. 2020). Interestingly, the side of the affected hindlimb in this model was not a fixed entity and was pharmacologically reversed. While the general opioid antagonist naloxone blocked
HL-PA formation, administration of selective $\kappa$-opioid antagonists to the rats with the right side CCI showing the left hindlimb flexion did not affect the asymmetry magnitude but reversed the flexion side; instead of the left limb the right limb became flexed (Watanabe et al. 2020). These findings suggest that there are components of sensorimotor and postural deficits, the development of which on the contra- vs. ipsilesional side is not determined by anatomical constrains but is controlled by a hitherto unknown neurohormonal mechanism that may be unraveled in the HL-PA model.

In the course of asymmetry analysis, we unexpectedly observed that both the left and right side CCI produced HL-PA with flexion of the same, (ie; left) hindlimb that was ipsi- and contralesional to the injured hemisphere, respectively. Besides HL-PA, the CCI rats may exhibit changes in body posture including body bending to the ipsilesional side. In contrast to the left side response in the HL-PA model, changes in body posture in rats with the left and right side CCI were mirror-symmetrical. This report presents these unanticipated HL-PA and body bending findings.

\section{Methods}

\section{Animals}

Adult male Sprague-Dawley rats (350-400 g body weight, purchased from Taconic, Denmark) housed in standard cage with food and water ad libitum and maintained under the $12 \mathrm{~h}$ ' light-dark cycle at a constant environmental temperature of $21^{\circ} \mathrm{C}$ (humidity: 65\%) were randomly assigned to experimental groups. Animals losing more than $10 \%$ body weight following the injury were excluded from the study. All procedures were approved by the research animal ethics board of Uppsala County (permits C101/13 and C165/14) and performed according to the rules and regulations of the Swedish Board of Agriculture.

\section{Surgery and histology}

\section{Controlled cortical impact (CCI)}

CCI was performed as previously described (Clausen et al. 2011; Watanabe et al. 2020). Briefly, a craniotomy, $5 \times 6 \mathrm{~mm}^{2}$, was centered at bregma $0.5 \mathrm{~mm}$ and $3.5 \mathrm{~mm}$ lateral to the midline over the right sensorimotor cortex. CCI brain injury was induced by a CCI-device (VCU Biomedical Engineering Facility, Richmond, Virginia, USA) using a $4.0 \mathrm{~mm}$ diameter piston, and producing a $1.0 \mathrm{~mm}$ compression of the brain at a speed of $2.4 \mathrm{~m} / \mathrm{s}$ and $100 \mathrm{~ms}$ duration. The impactor was perpendicular to the exposed cortex. For sham injury, animals underwent identical surgery and anesthesia without receiving the CCI. Animal weight was 
monitored and wound healing inspected daily post-surgery. No drugs were administered after brain surgery.

Three days following the CCI or sham injury, rats were sacrificed with overdose of pentobarbital and the brains were dissected. Frozen brains were cut into either $14 \mu \mathrm{m}$ or $35 \mu \mathrm{m}$ thick coronal sections. Following H\&E staining (Histolab, Gothenburg, Sweden) or Toluidine blue staining (Sigmma-Aldrich), images of the sections were acquired using a light microscope (Zeiss Stemi 2000-C; Zeiss Gmbh, Göttingen, Germany or Leica DM6000B, Leica Microsystems). The injury-induced loss of brain tissue was measured in H\&E stained section in each hemisphere with the SectionToVolume software (Hanell et al. 2012). The lesion area $\left(\mathrm{mm}^{2}\right)$ was calculated by outlining missing cortical tissue for each section taken at $0.5 \mathrm{~mm}$ intervals, and lesion volume $\left(\mathrm{mm}^{3}\right)$ determined by multiplying the sum of the contused areas obtained from each section by the distance between Sects. $(0.5 \mathrm{~mm})$. The tissue loss in the injured hemisphere after the left (mean \pm S.E.M.: $13.55 \pm 3.75 \mathrm{~mm}^{3}$; $n=5)$ and right $\left(13.03 \pm 4.14 \mathrm{~mm}^{3} ; n=5\right)$ CCI was virtually identical. Almost no tissue was lost in the contralateral hemisphere after the left $\left(0.02 \pm 0.00 \mathrm{~mm}^{3} ; n=5\right)$ and right $\left(0.01 \pm 0.00 \mathrm{~mm}^{3} ; n=5\right) \mathrm{CCI}$, and in both hemispheres after the left $\left(0.01 \pm 0.00 \mathrm{~mm}^{3} ; n=3\right)$ and right $\left(0.01 \pm 0.00 \mathrm{~mm}^{3}\right.$; $n=3$ ) sham injury. The architecture of the brain structures around the lesion site was visualized in [Fig. 1a-c (also see Fig. 1 in (Watanabe et al. 2020)]. The extent of the CCI injury ranged $2-4 \mathrm{~mm}$ rostrocaudally, $2-4 \mathrm{~mm}$ mediolaterally, and $1.5-2 \mathrm{~mm}$ in depth. No cortical injury was observed in sham-injured rats.

\section{Unilateral ablation brain injury (UBI)}

The scalp was incised and opened, a section of cranium located $0.5-4.0 \mathrm{~mm}$ posterior to the bregma and $1.8-3.8$ lateral to the midline on the left or right side was removed, and the part of the cerebral cortex located below the opening that includes the hindlimb representation area of the sensorimotor cortex was aspirated with a metallic pipette (tip diameter $0.5 \mathrm{~mm}$ ) (for details, see (Zhang et al. 2020)). For
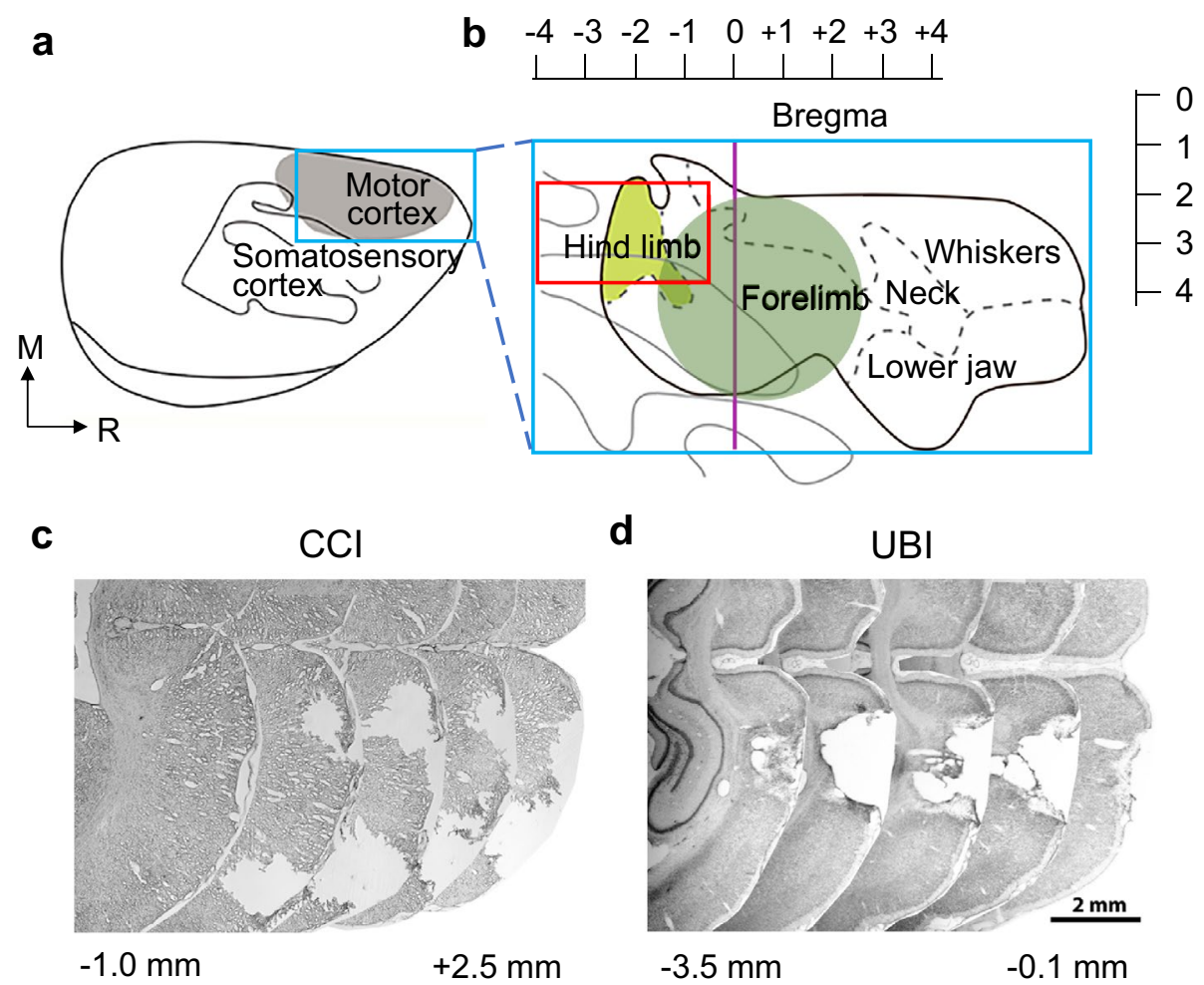

Fig. 1 Controlled cortical impact (CCI) and unilateral ablation brain injury (UBI) of the hindlimb motor cortex. a Schematic representation of the sensorimotor cortex of the rat brain (modified from (Tandon et al. 2008)). b Expanded cortex. Green circle denotes the intended lesion area although the actual lesion area slightly varied among the rats. Red rectangle denotes the intended lesion area although the actual lesion area slightly varied among the rats. Vertical purple line indicates the bregma plane. Scales above and on the right side indicate the distance in $\mathrm{mm}$ relative to the bregma rostro-

caudally and to the midline mediolaterally, respectively. c Five consecutive Nissl-stained cortical sections show the lesion site on the right cortex in two-dimension from a CCI rat. d Five consecutive Nissl-stained cortical sections show the lesion site on the right cortex in two-dimension from a UBI rat. Caudal is to the left and rostral is to the right for all the panels. The numbers below $\mathbf{c}$ and $\mathbf{d}$ indicate the position of the first and the last section in the figure relative to the bregma. Scale bar $=2 \mathrm{~mm}$ 
sham injury, animals underwent the same procedures, but the cortex was not ablated.

Localization and size of cortical lesions, and their comparison between the left and right side UBI rats were described in our previous papers (Watanabe et al. 2020; Zhang et al. 2020; Watanabe et al. 2021) (Fig. 1a-d in (Zhang et al. 2020)). Briefly, the brains were cut into $50 \mu \mathrm{m}$ think sections and stained with thionine. The lesion sites extended $3.2-5.0 \mathrm{~mm}$ rostrocaudally and $1.8-2.8 \mathrm{~mm}$ mediolaterally. The lesion was $1.0-1.5 \mathrm{~mm}$ in depth and did not affect the white matter below the cortex (Fig. 1a, b, d). The actual lesion size in some animals was slightly larger than intended (that was around $3.5 \times 2 \mathrm{~mm}$ ) due to tissue necrosis around the cavity border. The lesion volumes were not corrected for tissue shrinkage due to the fixation. The volumes were similar ( $P=0.84$, two tailed $t$ test) between the left (mean \pm S.E.M.: $\left.6.13 \pm 0.41 \mathrm{~mm}^{3} ; n=9\right)$ and right $\left(5.98 \pm 0.64 \mathrm{~mm}^{3} ; n=9\right)$ UBI rats.

\section{Analysis of the lateral body bending}

After administration of ketamine $(60 \mathrm{mg} / \mathrm{kg}$; intraperitoneally, i.p.), the rats were placed on the 1-mm grid paper in the prone position, the body was straightened in the rostro-caudal direction and then set free. The body posture was photographically recorded 10, 20 and 30 min after the injection and assessed as symmetric (not bent), or laterally bent to the left or to the right in the coronal plane. After the third measurement, additional dose of ketamine (10-20 mg/ $\mathrm{kg}$ ) supplemented with xylazine, an $\alpha_{2}$-adrenergic receptor agonist ( $5 \mathrm{mg} / \mathrm{kg}$; i.p.), was injected and 2-5 min later the body posture was photographically recorded. In a subset of rats, the first injection of ketamine was supplemented with xylazine $(5 \mathrm{mg} / \mathrm{kg}$; i.p.). Typical left and right bent rats are shown in Fig. 2. The body bending was analyzed on Day 3 or 4, and Day 7 after CCI or sham injury.

\section{Spinal cord transection}

The transection procedure was previously described (Watanabe et al. 2020, 2021; Zhang et al. 2020). Briefly, animals were anesthetized with sodium pentobarbital, and after measurement of postural asymmetry a laminectomy at the T2-T3 level was carried out. The spinal cord was doubly ligated and then completely transected between the ligatures. The completeness of the transection was confirmed by (i) inspecting the cord under microscope during the operation to ensure that no spared fibers bridged the transection site and that the rostral and caudal stumps of the spinal cord are completely retracted; (ii) placing a piece of Spongostan (Medispon ${ }^{\circledR}$ (MDD, Torun, Poland) between the rostral and caudal stumps of the spinal cord; and (iii) examining the spinal cord after termination of the experiment. In a subset a b
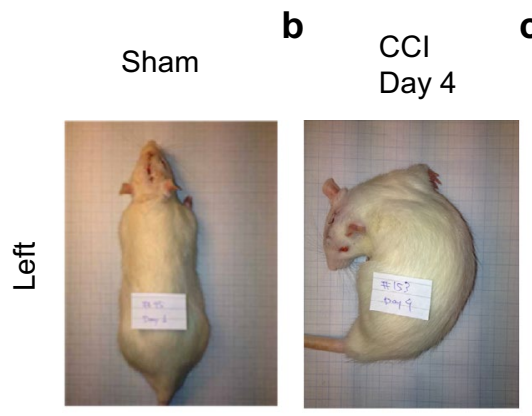

C

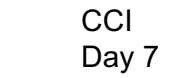

d
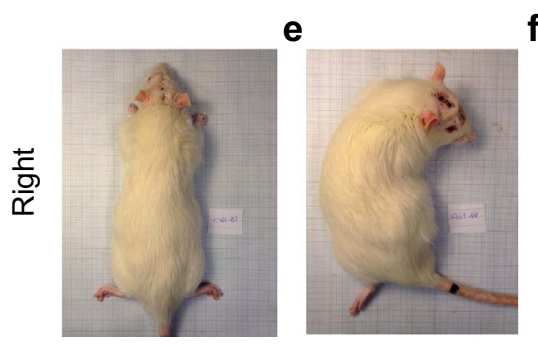

f
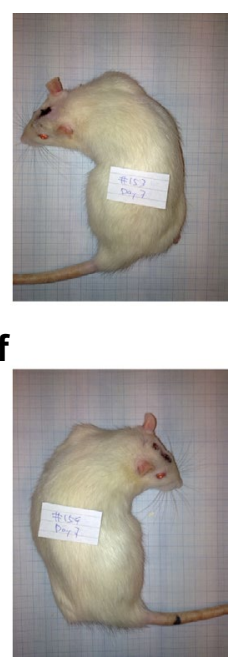

Fig. 2 The left and right CCI-induced body bending revealed under ketamine anesthesia. Rats with sham injury (a, d) or CCI (b, c, e, and f) were analyzed on Day $4(\mathbf{a}, \mathbf{b}, \mathbf{d}$ and $\mathbf{e})$ or Day 7 (c, f). CCI and sham surgery were performed either on the left $(\mathbf{a}-\mathbf{c})$ or $(\mathbf{d}-\mathbf{f})$ right side. Rats were categorized as symmetric (not bent), or laterally bent from the neck to the tail to the left or to the right in the coronal plane. The number of rats is shown in Table 1. All rats in each group displayed virtually the same either symmetric or asymmetric body posture at each of the 10, 20, and 30 min time points after ketamine administration

of rats, the 3-4-mm spinal cord segment was dissected and removed; the following microscopic and functional analyses demonstrated that the transection was complete, and that the brain injury-induced HL-PA was of the same size and direction as before the spinal transection (for details, see (Lukoyanov et al. 2020)).

\section{Analysis of HL-PA}

The magnitude of HL-PA and the side of the flexed limb were assessed as described previously (Bakalkin and Kobylyansky 1989; Watanabe et al. 2020; Zhang et al. 2020). Briefly, the measurements were performed under pentobarbital anesthesia $(60 \mathrm{mg} / \mathrm{kg}$, i.p.). The level of anesthesia was characterized by a barely perceptible corneal reflex and a lack of overall muscle tone. The rat was placed in the prone position on the 1-mm grid paper, and the hindlimbs were straightened in the hip and knee joints by gently pulling them backwards for 5-10 $\mathrm{mm}$ to reach the same level. Then, the limbs were set free and the magnitude of HL-PA was measured in millimeters as the length of the projection of the line connecting symmetric hindlimb distal points (digits 2-4) on the longitudinal axis of the rat (see Fig. 1e, f in (Watanabe et al. 2020)). The procedure was repeated six 
times in immediate succession. The limb displaying shorter projection was considered as flexed. The HL-PA magnitude in millimeters and the flexion side were used in statistical analysis. HL-PA was analyzed before and $60 \mathrm{~min}$ after spinal cord transection on Day 3 or Day 4 after CCI, UBI, or sham injury.

\section{Statistical analysis}

Processing and statistical analysis of HL-PA was performed after completion of the experiments by the statistician (D.S.), who was not involved in execution of experiments. Therefore, the results of intermediate statistical analyses could not affect acquisition of experimental data. The HL-PA magnitude in millimeters and the flexion side were analyzed using Bayesian regression models via full Bayesian framework by calling Stan 2.21 (Carpenter et al. 2017) from R 3.6.3 R Core Team using the brms 2.12 (Burkner 2017) interface. Predictors and outcomes were centered and scaled. To reduce the influence of outliers, models used Student's $t$ response distribution family with identity link function. Models had no intercepts with indexing approach to predictors (McElreath 2019). According to Stan recommendations (Stan Development Team 2019) weakly informative priors were used for group-level effects, residual SD and group-level SD. $P$ values, adjusted using the multivariate $t$ distribution with the same covariance structure as the estimates, were produced by frequentist summary in emmeans 1.4.6 (Searle et al. 1980). Medians of the posterior distribution and $95 \%$ highest posterior density continuous intervals were plotted. The contrast between groups was defined as significant if both $95 \%$ highest posterior density continuous intervals did not include zero and adjusted $P$ value was $\leq 0.05$.

\section{Results}

\section{CCl-induced body bending}

The effects of the unilateral CCI were analyzed in animals under ketamine, ketamine and xylazine, pentobarbital or isoflurane anesthesia. As reported previously (Hetzler and Wautlet 1985; Winters et al. 1986) administration of an anesthetic doses of ketamine produced a cataleptic immobility. Unexpectedly, the CCI rats injected with ketamine displayed strong consistent bending of the body from neck to tail to the left or to the right in the coronal plane (Fig. 2; Table 1). Intact rats and animals with the sham injury did not develop such asymmetric responses. The rats with the left CCI were bent to the left with the concave and convex on the left and right side, respectively. The effects of the right CCI were mirror symmetric to those of the left brain trauma. The bending response was evident at all three time points 10,20 and $30 \mathrm{~min}$ after ketamine administration, and was virtually the same on Day 4 and Day 7 after the CCI. All animals in each group demonstrated bending to virtually the same degree and direction (Fig. 2; Table 1). The body of the CCI rats was curved possibly due to muscle contraction on the concave side complemented by muscle stretching on the convex side that laterally flexed the spine. Administration of xylazine, an $\alpha_{2}$-adrenergic receptor agonist with analgesic and muscle relaxant properties, in combination with ketamine before the analysis, or after the third measurement of rats under ketamine anesthesia prevented and resolved the bending of the body, respectively. The bending was not observed in the CCI rats under pentobarbital or isoflurane anesthesia.
Table 1 The CCI effects on the body posture in the coronal plane analyzed under ketamine anesthesia

\begin{tabular}{llllll}
\hline Surgery and treatment & Surgery side & $\begin{array}{l}\text { The number } \\
\text { of rats }\end{array}$ & $\begin{array}{l}\text { Symmetric } \\
\text { rats }\end{array}$ & $\begin{array}{l}\text { Left bent rats } \\
\text { Right } \\
\text { bent } \\
\text { rats }\end{array}$ \\
\hline Intact rats & Left & 6 & 6 & 0 & 0 \\
Sham injury & Left & 6 & 3 & 0 & 0 \\
CCI & Left & 3 & 0 & 6 & 0 \\
CCI xylazine & Right & 4 & 3 & 0 & 0 \\
Sham injury & Right & 8 & 0 & 0 & 0 \\
CCI & & & 0 & 0 \\
\hline
\end{tabular}

The posture was assessed as symmetric (not bent), or laterally bent to the left or to the right in the coronal plane. All rats in a group were either symmetric or laterally bent at the 10, 20, and 30 min time point after ketamine administration. Xylazine co-administered with ketamine before the analysis $\left({ }^{\mathrm{a}}\right)$, or injected $30 \mathrm{~min}$ after ketamine resolved the CCI-induced asymmetry in all treated animals. The same bending pattern was evident on Day 4 and Day 7 after the CCI in each rat

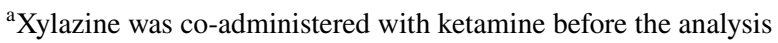




\section{The left CCI-induced HL-PA: ipsilesional flexion and spinal fixation}

We previously demonstrated that the right $\mathrm{CCI}$ along with the left and right UBI produced HL-PA with flexion of the contralateral hindlimb that was evident under pentobarbital or isoflurane anesthesia (Zhang et al. 2020; Watanabe et al. 2021). In the course of HL-PA analysis, we examined whether the left CCI also induced HL-PA, whether contralesional hindlimb was flexed, and whether HL-PA persisted after spinal cord transection. The HL-PA was analyzed under pentobarbital anesthesia before and $60 \mathrm{~min}$ after complete spinal cord transection at the T2-T3 level on Day 3 or Day 4 after the left CCI or sham injury (Fig. 3 and 4; Tables 2 and Online Resources 1-3). The right CCI rats, and the left and right UBI animals were analyzed for comparison. Differences between animal groups for the magnitude of postural asymmetry (MPA) and HL-PA size were assessed by Bayesian statistics. The left CCI rats developed HL-PA that was evident both before and after spinal cord transection, while

a

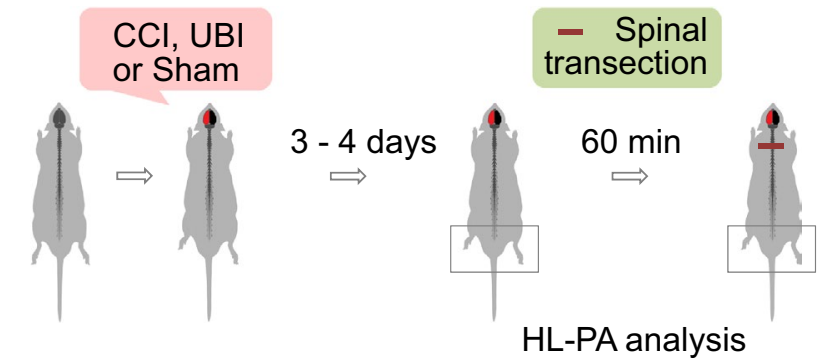

b

$$
\begin{aligned}
& \text { Spinal } \\
& \text { transection }
\end{aligned}
$$

C
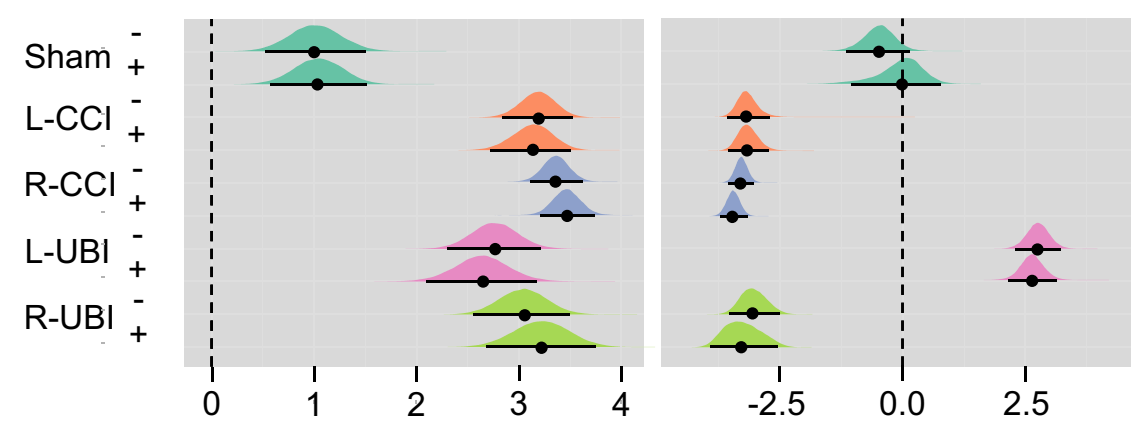

$$
\text { HL-PA magnitude (MPA), mm Left flexion } \begin{gathered}
\text { Right flexion } \\
\text { HL-PA, mm }
\end{gathered}
$$

d $\quad \begin{aligned} & \text { Spinal } \\ & \text { transection }\end{aligned}$

\section{e}
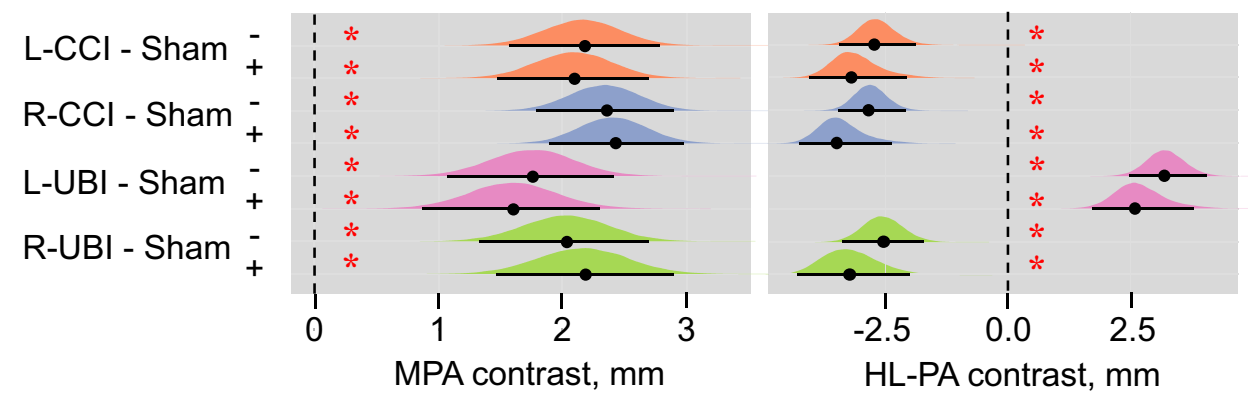

Fig. 3 The HL-PA induced by the left- (L-) or right- (R-) side CCI, and by the left- or right side UBI. a Experimental design. HL-PA was analyzed before [shown as (-)] and $60 \mathrm{~min}$ after [shown as (+)] spinal cord transection at the T2-3 level on the Day 3 or Day 4 after CCI, UBI or sham injury; the effects of the left CCI are shown. b, c The magnitude of HL-PA (MPA) and HL-PA size in millimeters (mm), respectively. In c, negative and positive HL-PA values are assigned to rats with the left and right hindlimb flexion, respectively. d, e Differences (contrasts) between the CCI and sham injury groups, and between the UBI and sham injury groups in the asymmetry magnitude and its size, respectively. The number of animals is given in Table 2. The MPA, HL-PA, and the contrasts are plotted as median (black circles), 95\% highest posterior density continuous intervals (black lines) and posterior density from Bayesian regression. *Significant differences (contrasts) between the groups: $95 \%$ highest posterior density continuous intervals did not include zero value, and adjusted $P$ values identified by Bayesian regression were $\leq 0.05$. Adjusted $P$ values are shown in Online Resource 1 and Online Resource 2 


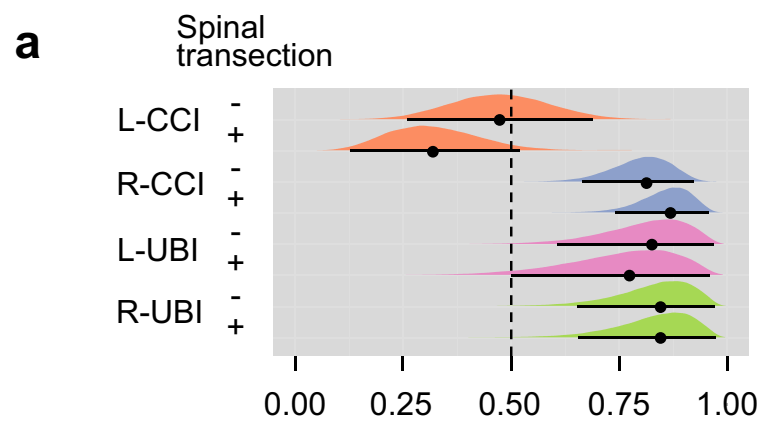

Probability of the contralesional flexion

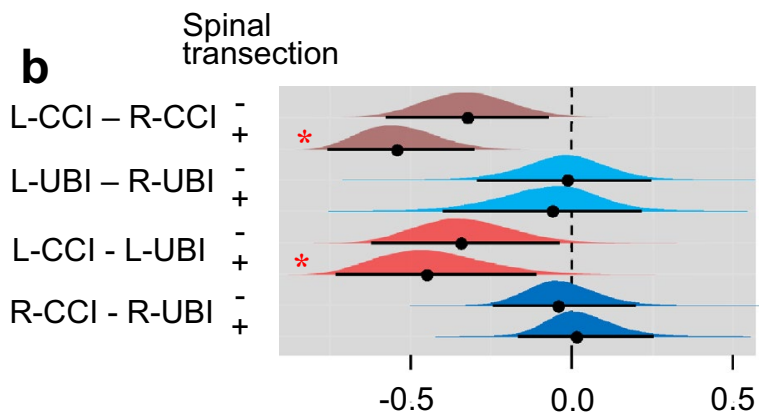

Contrast in the probability of contralesional flexion

Fig. 4 The probability of contralesional flexion in the left- (L-) and right- (R-) side CCI and the left- and right-side UBI groups. a The probability is plotted as median (black circles), 95\% highest posterior density continuous intervals (black lines) and posterior density from Bayesian regression. *Significant differences (contrasts) between the groups: $95 \%$ highest posterior density continuous intervals did not include zero value, and adjusted $P$ values identified by Bayesian regression were $\leq 0.05$. Adjusted $P$ values are shown in Online Resource 3. For details, see legend to Fig. 3

Table 2 The number of rats analyzed for development of HL-PA

\begin{tabular}{llr}
\hline Groups & \multicolumn{2}{l}{$\begin{array}{l}\text { Spinal transec- } \\
\text { tion }\end{array}$} \\
\cline { 2 - 3 } & Before & After \\
\hline Sham injury & 10 & 10 \\
L-CCI & 15 & 15 \\
R-CCI & 27 & 27 \\
L-UBI & 8 & 5 \\
R-UBI & 10 & 10 \\
\hline
\end{tabular}

HP-PA was not observed in sham-injured rats. HL-PA with similar MPA values was developed after the right CCI, and left and right UBI as reported previously (Zhang et al. 2020; Watanabe et al. 2021). The MPA in each of four groups that received brain injury was different from MPA in sham injury rats (Fig. 3b, d; Tables 2 and Online Resource 1). Consistent with the previous reports, the left hindlimb was flexed in the right $\mathrm{CCI}$ and right UBI rats, while the left UBI produced right flexion (Fig. 3c) (Zhang et al. 2020; Watanabe et al. 2021). Unexpectedly, and in contrast with these reports, rats with the left CCI developed left limb flexion (Fig. 3c, e; Online Resource 2). Effects of brain injuries in all four groups were different from those of sham injury with high significance. The HL-PA with virtually the same magnitude and direction were evident in rats analyzed before the spinal cord transection.

The probability to develop contralesional flexion was high in the right CCI and left and right UBI groups, while low in the left CCI rats (Fig. 4a). The left CCI group differed in this probability from the right CCI group and left UBI group after but not before spinal cord transection (Fig. 4b; Online Resource 3).

\section{Discussion}

The main finding of the present study was that the left and right CCI induced HL-PA with the left hindlimb flexion that was, respectively, ipsi- and contralateral to the injured hemisphere. Ipsilesional effects of a unilateral brain lesion, traumatic brain injury, and stroke were documented in animal experiments and clinical studies (Gonzalez et al. 2004; Varghese and Winstein 2019; Maenza et al. 2020). In stroke survivors, functional deficits of ipsilesional limb depended on two factors, namely the side of damage and severity of contralesional impairments (Varghese and Winstein 2019; Maenza et al. 2020). The most severe ipsilesional deficits were expressed in patients with left side stroke and severe contralesional impairments. Findings of this study corroborate these clinical observations by demonstration that there are components or signs of sensorimotor and postural deficits that are induced ipsilaterally by the left side brain lesion while the right side injury produces contralesional effects.

\section{Putative neural mechanism of HL-PA and body bending}

Neural mechanism may underlie the left side response to the left and right side CCI and could be mediated by the ipsiand contralateral descending pathways, respectively (Fig. 5). Different effects of the left and right CCI on corticospinal projecting neurons and descending pathways that signal to the ipsilateral spinal cord suggest that their anatomical organization differs between the left and right hemispheres. This neural asymmetry may be supported by human, rodent, and insect findings that demonstrated general lateralization of the motor system (Robinson 1979; Sainburg 2014; Knebel et al. 2018; Stancher et al. 2018; Zhang et al. 2020). At the same time, development of the contralesional hindlimb flexion induced by ablation injury to the left or right hemispheres [Fig. 5c and (Lukoyanov et al. 2020; Watanabe et al. 
Neural

mechanism

a

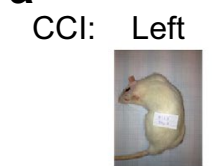

b

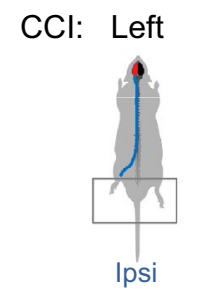

C
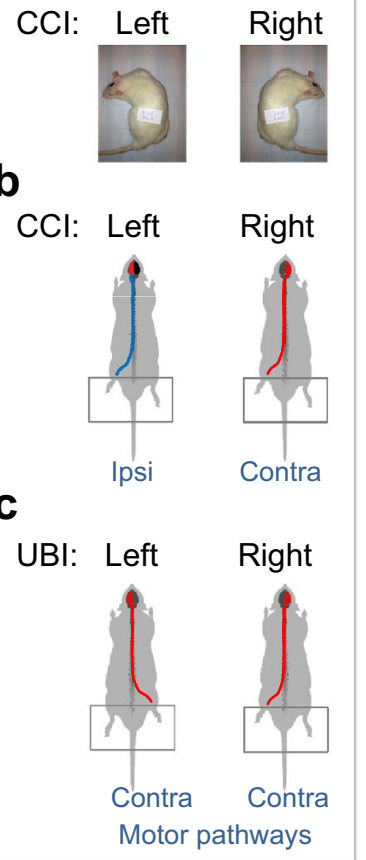

Right

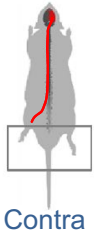

Contra
Neurohormonal mechanism

Postural asymmetry inducing factors (PAFs)

d

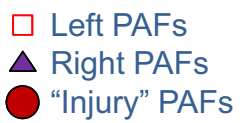

CCl:
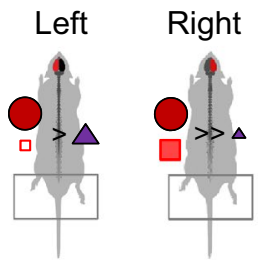

e

UBI:
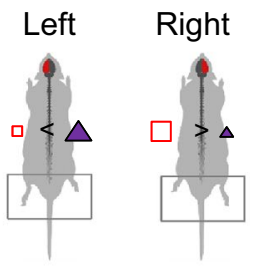

Fig. 5 Hypothetical neural and neurohormonal mechanisms of contralesional and ipsilesional responses induced by the left and right $\mathrm{CCI}$, and the left and right UBI. a-c Descending motor tracts may mediate effects of the brain injury on body bending and HL-PA. a Lateral body bending directed to the ipsilesional side after the leftand right-side $\mathrm{CCI}$, and $\mathbf{c}$ formation of the contralesional hindlimb flexion after the left- and right-side UBI may be mediated by decussating neural tracts that are mirror-symmetrical. b Ipsilateral and decussating contralateral motor pathways may be engaged in formation of HL-PA with left hindlimb flexion after the left- and rightside CCI, respectively; the left CCI in contrast to the left UBI and right CCI may activate the ipsilateral motor pathway. This pathway may control hindlimb responses but not body bending, and may be anatomically asymmetric because the right CCI instead produces the contralesional hindlimb flexion. d, e Two groups of neurohormones and neuropeptides may produce HL-PA with flexion of the left or right hindlimb, respectively (Bakalkin et al. 1989; Watanabe et al. 2020, 2021). Activity of these left and right postural asymmetry inducing factors (PAFs) may be balanced in the symmetric brain, while CCI and UBI may impair the balance and shift an equilibrium in PAFs activity to favor the factors producing either the contralesional or ipsilesional hindlimb response. Some of the PAFs, for example, endogenous opioid peptides dynorphins and Met-enkephalin are involved in tissue injury, pain and stress, and overproduced after brain trauma and spinal cord injury (Hauser et al. 2005; Hussain et al. 2012; Maximyuk et al. 2015; Smeets et al. 2015). The overproduction or excessive release of these injury-associated PAFs may be similar after the left and right side CCI. Similarly with synthetic dynorphins and Met-enkephalin that induce hindlimb flexion on the left side in animals with intact brain, the endogenous injury-associated PAFs may induce a unilateral response, namely the left hindlimb flexion (Bakalkin et al. 1989; Watanabe et al. 2020). In comparison with the CCI, the UBI does not produce a severe brain damage and, therefore, may not activate the injury-associated PAFs, while the side-specific PAFs may be generated and enable formation of the contralesional hindlimb flexion (Bakalkin et al. 1989; Watanabe et al. 2020)

2020, 2021; Zhang et al. 2020)] suggests that the ipsilateral descending motor pathways are less affected by the UBI $v s$. the CCI in the left hemisphere.

In contrast to the left side hindlimb response developed under pentobarbital or isoflurane anesthesia, the left and right $\mathrm{CCI}$ rats displayed the mirror-symmetrical body bending patterns under ketamine anesthesia, suggesting that the injury effects were mediated through mirror symmetric neural pathways. To our knowledge the brain injury-induced postural effects that were not seen in unanesthetized animals but uncovered by administration of ketamine, have not yet been reported. Bulbospinal tracts specifically reticulospinal and vestibulospinal tracts innervate the ipsilateral axial and proximal limb muscles controlling posture and balance (Purves et al. 2012). Both tracts receive projections from sensorimotor cortex (McCall et al. 2017; Cregg et al. 2020), and may be affected by CCI and therefore their activity may be reduced on the contralesional side.

Ketamine, a non-competitive $N$-methyl-D-aspartate receptor antagonist, may interfere with synaptic transmission in these tracts that use glutamate as a neurotransmitter. The contra- and ipsilateral tracts may be differentially affected by the CCI and develop different sensitivity to ketamine. Their different inhibition by ketamine may result in stronger contraction of ipsi- $v s$. contralesional axial muscles leading to body bending. Alternatively, stimulation of the $\mathrm{N}$-methylD-aspartate ion channel by endogenous glutamate may have a compensatory function and maintain a balance in activity of the affected and unaffected counterparts of mirrorsymmetrical neural circuits in unanesthetized animals after a unilateral brain trauma. This could counteract the lateral deformation of the body, while inhibition of this mechanism by ketamine would allow the body bending in rats with CCI. Overall, the left and right CCI produced the mirrorsymmetrical effects on the body bending, while the left side flexion was revealed in the HL-PA model and was apparently mediated by different neural circuits.

The asymmetric tonic neck reflex (ATNR) discovered by Magnus and de Kleijn is a primitive reflex that is present early in life and may reemerge in adults after brain injury (Shevell 2009). It was described as a "fencing" posture where rotation of the head results in an asymmetric muscle contraction pattern with extension of the limbs ipsilateral to the direction of head rotation, and flexion of the limbs contralateral to the direction of head rotation. In recent studies, evidence was provided for the re-emergence of ATNR after stroke and traumatic brain injury, and for its reliance on ipsilateral reticulospinal pathways as the mechanism (Ellis et al. 2012; McPherson et al. 2018). At the same time, a unilateral microstimulation of the reticular formation produced head movements that were directed ipsilaterally to both the side of stimulation and the side of activation of the upper limb flexors (Drew and Rossignol 1990; Quessy 
and Freedman 2004). In this experimental setting, the direction of head rotation was opposite to the ATNR. The effects induced by ketamine administration to the CCI rats resembled the postural pattern produced by microsimulation of the reticular formation in pairing of the head rotation to the ipsilesional side with bending of the body from neck to tail with the concave on the same side. This postural pattern may be induced through asymmetric effects of ketamine on the contra- and ipsilesional reticulospinal pathways differently affected by the CCI. The link of the ketamine effects with the ATNR requires further analysis. Formation of HL-PA was not apparently related to the ATNR. First, because the head was fixed in the symmetric position in these experiments, and second, because HL-PA persisted after complete spinal cord transection. Nonetheless, it would be interesting to examine whether the head rotation to or from the affected limb would produce any changes in the HL-PA.

\section{Side-specific neuroendocrine mechanism of HL-PA formation}

The CCI and UBI differ in their pathophysiological mechanisms. CCI produces substantial and progressive injury to the white matter and underlying structures (Hall et al. 2005, 2008). In contrast, the lesion caused by the UBI is confined to the cortex. In the CCI, brain damage is caused by mechanical stress-induced axonal injury and vascular dysfunction followed by dispersed neuronal degeneration and death, neuroinflammation, and excitotoxicity (Hall et al. 2005, 2008; $\mathrm{Ng}$ and Lee 2019; Lacalle-Aurioles et al. 2020). These processes are mediated by a variety of endogenous regulatory molecules including neuropeptides, cytokines, and neurotrophic factors. These molecules may also shape functional connectivity within neural circuits and modify their functions (Bargmann 2012; Nusbaum et al. 2017). These injuryassociated molecules may be activated by both the left or right side brain injury, while in intact brain some of them may be involved in lateralized processes and even produce side-specific effects (Deliagina et al. 2000; Hussain et al. 2012; Marlin et al. 2015; Nation et al. 2018; Phelps et al. 2019; Watanabe et al. 2020, 2021). For example, the opioid peptides dynorphins are pathogenic when they are overexpressed in response to chronic pain, and brain, and spinal cord injury (Caudle and Mannes 2000; Hauser et al. 2005; Hussain et al. 2012; Maximyuk et al. 2015; Smeets et al. 2015). Upregulated dynorphins may also induce asymmetric responses including development of HL-PA (Bakalkin and Kobylyansky 1989; Watanabe et al. 2020, 2021). We previously demonstrated that administration of opioid antagonists to rats with a unilateral brain lesion inhibited HL-PA formation, while opioid peptides and synthetic opioids mimicked effects of brain injury by inducing HL-PA in rats with intact brain (Chazov et al. 1981; Bakalkin et al. 1986; Bakalkin and Kobylyansky 1989; Watanabe et al. 2020, 2021). The right UBI-induced left flexion may be mediated by dynorphins and Met-enkephalin, while the left UBI-induced HL-PA with right flexion may be mediated by Leu-enkephalin (Chazov et al. 1981; Bakalkin et al. 1986; Bakalkin and Kobylyansky 1989; Watanabe et al. 2020, 2021).

In the context of the HL-PA neurohormonal mechanism (Fig. 5d, e), the left side response to the left and right side CCI may be developed due to overproduction or excessive release of pathogenic dynorphins, Met-enkephalin or other neurohormones with the left side actions (Fig. 5d). The "left side" neurohormones may prevail over the "right side" regulatory factors after both the left and right CCI but not after UBI. The UBI mechanistically differs from the CCI and may not activate these neurohormonal systems at least to the same extent. Neural pathways or neurohormonal systems that are differently activated by the left and right UBI may dominate in signaling from the injured brain to the lumbar neural circuits controlling the right and left hindlimb muscles, respectively (Fig. 5e).

\section{Limitations}

The limitations of this study were first that the CCI effects were examined in anesthetized animals. Still, the analysis was valid; anesthesia with isoflurane and pentobarbital did not interfere with HL-PA magnitude and flexion side as it was evident from analyses of anesthetized animals and unanesthetized decerebrate rats in our previous studies (Lukoyanov et al. 2020; Zhang et al. 2020). Second, the study focused on the HL-PA and body bending while did not evaluate pathophysiological and neuroendocrine mechanisms of postural deficits. However, the HL-PA has been validated in our previous studies by analysis of the hindlimb resistance to stretch, hindlimb withdrawal reflexes evoked by electrical stimulation and recorded with EMG technique, and hindlimb motor deficits in locomotor tasks in rats with UBI (Lukoyanov et al. 2020; Zhang et al. 2020; Watanabe et al. 2021). The contra- and ipsilesional hindlimbs displayed different musculo-articular resistance to stretch and different reflex activation patterns after the brain injury. The direction of the UBI-induced effects perfectly correlated among the HL-PA, the stretching resistance and EMG asymmetries, and asymmetric hindlimb motor impairments in locomotor tasks. Also, the HL-PA magnitude and the contra-ipsilesional differences in the stretching resistance strongly correlated. We did not identify the afferent and efferent neural circuits involved, and neural and neurohormonal pathways that signal from the injured brain to spinal cord. On the other hand we demonstrated that the HL-PA modelled several pathophysiological features of the upper motor neuron syndrome induced by traumatic brain injury or stroke primarily their asymmetric patterns (Lukoyanov et al. 2020; 
Zhang et al. 2020; Watanabe et al. 2021). Furthermore, we showed the HL-PA was blocked by a muscle relaxant while the asymmetry was resistant to bilateral lumbar deafferentation suggesting the mechanism dependent on the efferent drive but not on afferent input (Zhang et al. 2020). In this instance, HL-PA is arguably similar to "spastic dystonia", a tonic muscle overactivity that contributes to "hemiplegic posture" in patients with stroke or cerebral palsy (Gracies 2005; Sheean and McGuire 2009; Lorentzen et al. 2018).

\section{Conclusions}

This study describes an unusual left-side hindlimb response to a unilateral traumatic brain injury that does not depend on the side of the lesion. The mirror symmetry was disrupted in the development of HL-PA that was induced by traumatic injury of either the left or right hemisphere. At the same time, mirror-symmetrical postural responses were developed after the localized ablation cortical injury in the HL-PA model, and after the CCI in body bending. Lateralized neural and side-specific neurohormonal mechanisms may signal from an injured hemisphere to spinal neural circuits and account for the disrupted mirror symmetry in effects of the left and right side CCI. These mechanisms may have a role in development of the ipsilesional $v s$. contralesional sensorimotor and postural deficits secondary to traumatic brain injury and stroke.

Supplementary Information The online version contains supplementary material available at https://doi.org/10.1007/s00221-021-06118-4.

Acknowledgements We are grateful to Dr. Michael Ossipov for discussion and manuscript processing.

Funding Open access funding provided by Uppsala University. The study was supported by the Swedish Research Council (Grants K201462X-12190-19-5 and 2019-01771-3) to G.B., L. Hiertas Minne foundation, and P.O. Zetterling foundation to O.N., Uppsala University to G.B.

Data availability Data supporting the findings of this study are available within the article, its Online Resources, or upon request.

\section{Declarations}

Conflict of interest Authors report no conflict of interest.

Open Access This article is licensed under a Creative Commons Attribution 4.0 International License, which permits use, sharing, adaptation, distribution and reproduction in any medium or format, as long as you give appropriate credit to the original author(s) and the source, provide a link to the Creative Commons licence, and indicate if changes were made. The images or other third party material in this article are included in the article's Creative Commons licence, unless indicated otherwise in a credit line to the material. If material is not included in the article's Creative Commons licence and your intended use is not permitted by statutory regulation or exceeds the permitted use, you will need to obtain permission directly from the copyright holder. To view a copy of this licence, visit http://creativecommons.org/licenses/by/4.0/.

\section{References}

Bakalkin G, Kobylyansky AG (1989) Opioids induce postural asymmetry in spinal rat: the side of the flexed limb depends upon the type of opioid agonist. Brain Res 480:277-289. https://doi.org/10. 1016/0006-8993(89)90193-5

Bakalkin G, Kobylyansky AG, Nagornaya LV, Yarygin KN, Titov MI (1986) Met-enkephalin-induced release into the blood of a factor causing postural asymmetry. Peptides 7:551-556. https://doi.org/ 10.1016/0196-9781(86)90025-2

Bakalkin G, Pivovarov A, Kobylyansky AG, Yarygin KN, Akparov V (1989) Ipsilateral responses induced by factors present in left and right hemispheres. Int J Neurosci 47:217-230

Bargmann CI (2012) Beyond the connectome: how neuromodulators shape neural circuits. BioEssays 34:458-465. https://doi.org/10. 1002/bies.201100185

Burkner PC (2017) brms: an R package for Bayesian multilevel models using stan. Stat Softw 80:1-28. https://doi.org/10.18637/jss. v080.i01

Carpenter BL, Liu J, Qi L, Wang C, O'Connor KL (2017) Integrin alpha6beta4 upregulates amphiregulin and epiregulin through base excision repair-mediated DNA demethylation and promotes genome-wide DNA hypomethylation. Sci Rep 7:6174. https://doi. org/10.1038/s41598-017-06351-4

Caudle RM, Mannes AJ (2000) Dynorphin: friend or foe? Pain 87:235239. https://doi.org/10.1016/s0304-3959(00)00360-2

Chamberlain TJ, Halick P, Gerard RW (1963) Fixation of experience in the rat spinal cord. J Neurophysiol 26:662-673. https://doi.org/ 10.1152/jn. 1963.26.4.662

Chazov EI, Bakalkin G, Yarigin KN, Trushina ED, Titov MI, Smirnov VN (1981) Enkephalins induce asymmetrical effects on posture in the rat. Experientia 37:887-889. https://doi.org/10.1007/bf019 85696

Clausen F, Hanell A, Israelsson C et al (2011) Neutralization of interleukin-1beta reduces cerebral edema and tissue loss and improves late cognitive outcome following traumatic brain injury in mice. Eur J Neurosci 34:110-123. https://doi.org/10.1111/j.1460-9568. 2011.07723.x

Coelho DB, Fernandes CA, Martinelli AR, Teixeira LA (2019) Right in comparison to left cerebral hemisphere damage by stroke induces poorer muscular responses to stance perturbation regardless of visual information. J Stroke Cerebrovasc Dis 28:954-962. https:// doi.org/10.1016/j.jstrokecerebrovasdis.2018.12.021

Cregg JM, Leiras R, Montalant A, Wanken P, Wickersham IR, Kiehn O (2020) Brainstem neurons that command mammalian locomotor asymmetries. Nat Neurosci 23:730-740. https://doi.org/10.1038/ s41593-020-0633-7

de Paiva Silva FP, Freitas S, Banjai RM, Alouche SR (2018) Ipsilesional arm aiming movements after stroke: influence of the degree of contralesional impairment. J Mot Behav 50:104-115. https:// doi.org/10.1080/00222895.2017.1306479

Deliagina TG, Orlovsky GN, Selverston AI, Arshavsky YI (2000) Asymmetrical effect of GABA on the postural orientation in Clione. J Neurophysiol 84:1673-1676. https://doi.org/10.1152/ jn.2000.84.3.1673

Drew T, Rossignol S (1990) Functional organization within the medullary reticular formation of intact unanesthetized cat. I. movements evoked by microstimulation. J Neurophysiol 64:767-781. https:// doi.org/10.1152/jn.1990.64.3.767

Ellis MD, Drogos J, Carmona C, Keller T, Dewald JP (2012) Neck rotation modulates flexion synergy torques, indicating an ipsilateral 
reticulospinal source for impairment in stroke. J Neurophysiol 108:3096-3104. https://doi.org/10.1152/jn.01030.2011

Fernandes CA, Coelho DB, Martinelli AR, Teixeira LA (2018) Right cerebral hemisphere specialization for quiet and perturbed body balance control: evidence from unilateral stroke. Hum Mov Sci 57:374-387. https://doi.org/10.1016/j.humov.2017.09.015

Stan Development Team (2019) Stan modeling language users guide and reference manual, 2.21. https://mc-stan.org ((Prior choice recommendations. Retrieved 2 May 2019 from https://github. $\mathrm{com} / \mathrm{stan}$-dev/stan/wiki/Prior-Choice-Recommendations)

Gonzalez CL, Gharbawie OA, Williams PT, Kleim JA, Kolb B, Whishaw IQ (2004) Evidence for bilateral control of skilled movements: ipsilateral skilled forelimb reaching deficits and functional recovery in rats follow motor cortex and lateral frontal cortex lesions. Eur J Neurosci 20:3442-3452. https://doi.org/ 10.1111/j.1460-9568.2004.03751.x

Gracies JM (2005) Pathophysiology of spastic paresis. I: paresis and soft tissue changes. Muscle Nerve 31:535-551. https://doi.org/ 10.1002/mus.20284

Hall ED, Sullivan PG, Gibson TR, Pavel KM, Thompson BM, Scheff SW (2005) Spatial and temporal characteristics of neurodegeneration after controlled cortical impact in mice: more than a focal brain injury. J Neurotrauma 22:252-265. https://doi.org/ 10.1089/neu.2005.22.252

Hall ED, Bryant YD, Cho W, Sullivan PG (2008) Evolution of posttraumatic neurodegeneration after controlled cortical impact traumatic brain injury in mice and rats as assessed by the de Olmos silver and fluorojade staining methods. J Neurotrauma 25:235-247. https://doi.org/10.1089/neu.2007.0383

Hanell A, Hedin J, Clausen F, Marklund N (2012) Facilitated assessment of tissue loss following traumatic brain injury. Front Neurol 3:29. https://doi.org/10.3389/fneur.2012.00029

Hanna-Pladdy B, Mendoza JE, Apostolos GT, Heilman KM (2002) Lateralised motor control: hemispheric damage and the loss of deftness. J Neurol Neurosurg Psychiatry 73:574-577. https:// doi.org/10.1136/jnnp.73.5.574

Harris JE, Eng JJ (2006) Individuals with the dominant hand affected following stroke demonstrate less impairment than those with the nondominant hand affected. Neurorehabil Neural Repair 20:380-389. https://doi.org/10.1177/1545968305284528

Hauser KF, Aldrich JV, Anderson KJ et al (2005) Pathobiology of dynorphins in trauma and disease. Front Biosci 10:216-235. https://doi.org/10.2741/1522

Hetzler BE, Wautlet BS (1985) Ketamine-induced locomotion in rats in an open-field. Pharmacol Biochem Behav 22:653-655. https://doi.org/10.1016/0091-3057(85)90291-6

Hultborn H, Malmsten J (1983) Changes in segmental reflexes following chronic spinal cord hemisection in the cat. II. Conditioned monosynaptic test reflexes. Acta Physiol Scand 119:423433. https://doi.org/10.1111/j.1748-1716.1983.tb07358.x

Hussain ZM, Fitting S, Watanabe $\mathrm{H}$ et al (2012) Lateralized response of dynorphin a peptide levels after traumatic brain injury. J Neurotrauma 29:1785-1793. https://doi.org/10.1089/neu.2011.2286

Knebel D, Rillich J, Ayali A, Pfluger HJ, Rigosi E (2018) Ex vivo recordings reveal desert locust forelimb control is asymmetric. Curr Biol 28:R1290-R1291. https://doi.org/10.1016/j.cub.2018. 09.063

Lacalle-Aurioles M, Cassel de Camps C, Zorca CE, Beitel LK, Durcan TM (2020) Applying hiPSCs and biomaterials towards an understanding and treatment of traumatic brain injury. Front Cell Neurosci 14:594304. https://doi.org/10.3389/fncel.2020. 594304

Lorentzen J, Pradines M, Gracies JM, Bo Nielsen J (2018) On DennyBrown's 'spastic dystonia' - what is it and what causes it? Clin Neurophysiol 129:89-94. https://doi.org/10.1016/j.clinph.2017. 10.023
Lukoyanov N, Watanabe H, Carvalho LS et al (2020) Endocrine signaling mediates asymmetric motor deficits after unilateral brain injury. BioRxiv. https://doi.org/10.1101/2020.04.23.056937

Maenza C, Good DC, Winstein CJ, Wagstaff DA, Sainburg RL (2020) Functional deficits in the less-impaired arm of stroke survivors depend on hemisphere of damage and extent of paretic arm impairment. Neurorehabil Neural Repair 34:39-50. https://doi. org/10.1177/1545968319875951

Mani S, Mutha PK, Przybyla A, Haaland KY, Good DC, Sainburg RL (2013) Contralesional motor deficits after unilateral stroke reflect hemisphere-specific control mechanisms. Brain 136:1288-1303. https://doi.org/10.1093/brain/aws283

Marlin BJ, Mitre M, D’Amour JA, Chao MV, Froemke RC (2015) Oxytocin enables maternal behaviour by balancing cortical inhibition. Nature 520:499-504. https://doi.org/10.1038/nature14402

Maximyuk O, Khmyz V, Lindskog CJ et al (2015) Plasma membrane poration by opioid neuropeptides: a possible mechanism of pathological signal transduction. Cell Death Dis. https://doi.org/10. 1038/cddis.2015.39

McCall AA, Miller DM, Yates BJ (2017) Descending influences on vestibulospinal and vestibulosympathetic reflexes. Front Neurol 8:112. https://doi.org/10.3389/fneur.2017.00112

McElreath R (2019) Statistical rethinking. A Bayesian course with examples in R and Stan. Chapman and Hall/CRC

McPherson JG, Chen A, Ellis MD, Yao J, Heckman CJ, Dewald JPA (2018) Progressive recruitment of contralesional cortico-reticulospinal pathways drives motor impairment post stroke. J Physiol 596:1211-1225. https://doi.org/10.1113/JP274968

Nation KM, De Felice M, Hernandez PI, Dodick DW, Neugebauer V, Navratilova E, Porreca F (2018) Lateralized kappa opioid receptor signaling from the amygdala central nucleus promotes stressinduced functional pain. Pain 159:919-928. https://doi.org/10. 1097/j.pain.0000000000001167

Ng SY, Lee AYW (2019) Traumatic brain injuries: pathophysiology and potential therapeutic targets. Front Cell Neurosci 13:528. https://doi.org/10.3389/fncel.2019.00528

Nusbaum MP, Blitz DM, Marder E (2017) Functional consequences of neuropeptide and small-molecule co-transmission. Nat Rev Neurosci 18:389-403. https://doi.org/10.1038/nrn.2017.56

Phelps CE, Navratilova E, Dickenson AH, Porreca F, Bannister K (2019) Kappa opioid signaling in the right central amygdala causes hind paw specific loss of diffuse noxious inhibitory controls in experimental neuropathic pain. Pain 160:1614-1621. https://doi.org/10.1097/j.pain.0000000000001553

Purves D, Augustine GJ, Fitzpatrick D, Hall WC, LaMantia AS, White LE (2012) Neuroscience. Sinauer Associates

Quessy S, Freedman EG (2004) Electrical stimulation of rhesus monkey nucleus reticularis gigantocellularis. I. Characteristics of evoked head movements. Exp Brain Res 156:342-356. https:// doi.org/10.1007/s00221-003-1787-8

Robinson RG (1979) Differential behavioral and biochemical effects of right and left hemispheric cerebral infarction in the rat. Science 205:707-710. https://doi.org/10.1126/science.462179

Rossignol S, Frigon A (2011) Recovery of locomotion after spinal cord injury: some facts and mechanisms. Annu Rev Neurosci 34:413440. https://doi.org/10.1146/annurev-neuro-061010-113746

Sainburg RL (2014) Convergent models of handedness and brain lateralization. Front Psychol 5:1092. https://doi.org/10.3389/fpsyg. 2014.01092

Searle SR, Speed FM, Milliken GA (1980) Population marginal means in the linear model: an alternative to least squares means. Am Stat 34:216-221. https://doi.org/10.1080/00031305.1980.10483031

Sheean G, McGuire JR (2009) Spastic hypertonia and movement disorders: pathophysiology, clinical presentation, and quantification. PM R 1:827-833. https://doi.org/10.1016/j.pmrj.2009.08.002 
Shevell M (2009) The tripartite origins of the tonic neck reflex: gesell, gerstmann, and magnus. Neurology 72:850-853. https://doi.org/ 10.1212/01.wnl.0000343961.35429.09

Smeets CJ, Jezierska J, Watanabe H et al (2015) Elevated mutant dynorphin a causes Purkinje cell loss and motor dysfunction in spinocerebellar ataxia type 23. Brain 138:2537-2552. https://doi. org/10.1093/brain/awv195

Stancher G, Sovrano VA, Vallortigara G (2018) Motor asymmetries in fishes, amphibians, and reptiles. Prog Brain Res 238:33-56. https://doi.org/10.1016/bs.pbr.2018.06.002

Tandon S, Kambi N, Jain N (2008) Overlapping representations of the neck and whiskers in the rat motor cortex revealed by mapping at different anaesthetic depths. Eur J Neurosci 27:228-237. https:// doi.org/10.1111/j.1460-9568.2007.05997.x

Varghese R, Winstein CJ (2019) Relationship between motor capacity of the contralesional and ipsilesional hand depends on the side of stroke in chronic stroke survivors with mild-to-moderate impairment. Front Neurol 10:1340. https://doi.org/10.3389/fneur.2019. 01340

Varlinskaia EI, Rogachii MG, Klement'ev BI, Vartanian GA (1984) Dynamics of the activity of the postural asymmetry factor after unilateral damage to the motor area of the cortex. Biull Eksp Biol Med 98:281-283

Watanabe H, Nosova O, Sarkisyan D et al (2020) Ipsilesional versus contralesional postural deficits induced by unilateral brain trauma: a side reversal by opioid mechanism. Brain Commun. https://doi. org/10.1093/braincomms/fcaa208

Watanabe H, Nosova O, Sarkisyan D et al (2021) Left-right side-specific neuropeptide mechanism mediates contralateral responses to a unilateral brain injury. eNeuro. https://doi.org/10.1523/ ENEURO.0548-20.2021

Winters WD, Hance AJ, Cadd GC, Lakin ML (1986) Seasonal and sex influences on ketamine-induced analgesia and catalepsy in the rat; a possible role for melatonin. Neuropharmacology 25:1095-1101. https://doi.org/10.1016/0028-3908(86)90156-5

Zhang M, Watanabe H, Sarkisyan D et al (2020) Hindlimb motor responses to unilateral brain injury: spinal cord encoding and left-right asymmetry. Brain Commun. https://doi.org/10.1093/ braincomms/fcaa055

Publisher's Note Springer Nature remains neutral with regard to jurisdictional claims in published maps and institutional affiliations. 